\title{
VALORACIÓN Y ANÁLISIS DE INDICADORES DE SOSTENIBILIDAD EN SEIS UNIDADES DE PRODUCCIÓN AGROPECUARIA DE LA CUENCA MEDIA DEL RÍO CHINCHINÁ
}

\author{
Paola Andrea Calderón Cuartas ${ }^{1}$ \& Gloria Yaneth Flórez Yepes ${ }^{2}$
}

Recibido el 31 de marzo de 2014, aprobado el 22 de agosto de 2014 y actualizado el 27 de abril de 2015

DOI: 10.17151/luaz.2015.41.5

\section{RESUMEN}

En la cuenca media del río Chinchiná se seleccionaron seis unidades de producción agropecuaria con aplicación de estrategias de producción más limpia, con el fin de conocer el nivel de sostenibilidad para cada una, adaptando la metodología de indicadores de sostenibilidad propuesta por ASPROINCA (Arango, 2003). Las variables medidas fueron: seguridad alimentaria, mercado, dependencia de insumos externos, mano de obra, conservación de agua, de suelo y de bosque, nivel de complejidad del sistema, nivel de reciclaje, nivel de integración de subsistemas, compromiso familiar, formas organizativas de trabajo y valores ambientales. El gráfico tipo redegrama generado por el ejercicio de valoración y análisis de dichos indicadores, le permite al propietario del predio reconocer las debilidades y fortalezas de su unidad productiva respecto a temas económicos, ecológicos y socioculturales, identificando puntos críticos para la priorización de actividades de sostenibilidad. Los resultados demuestran que cada unidad productiva tiene aspectos particulares respecto al uso de recursos. Los aspectos que afectan actualmente la sostenibilidad de los predios estudiados fueron la alta dependencia de insumos externos y la baja competitividad en los mercados. Los valores ambientales y la conservación de recursos naturales, agua, suelo y bosque, son factores positivos característicos de las unidades productivas que participaron en el proyecto.

\section{PALABRAS CLAVE}

Variables ecológicas, variables económicas, variables socioculturales, producción más limpia, sostenibilidad.

\section{ASSESSMENT AND SUSTAINABILITY INDICATORS ANALYSIS OF SIX AGRICULTURAL PRODUCTION UNITS IN THE MIDDLE BASIN OF THE CHINCHINÁ RIVER}

\section{ABSTRACT}

Six agricultural production units applying cleaner production strategies were selected in the middle basin of the Chinchina river (Caldas, Colombia), in order to establish each unit sustainability level, adapting the sustainability indicators methodology proposed by ASPROINCA (Arango, 2003). The measured variables were: 
food security, markets, dependence on external supplies, labor, conservation of water, soil and forest, level of the system complexity, level of recycling, level of subsystems integration, family commitment, organizational forms of work and environmental values. The network type pictogram generated through the assessment and analysis exercise of such indicators allows the farmland owner to recognize the strengths and weaknesses of his production unit regarding economic, ecologic and socio-cultural issues, and to identify critical points for prioritization of sustainability activities. The obtained results show that each production unit has distinctive features about resources usage...The main aspects with mayor impact on sustainability of the studied farmlands were the high dependency of external supplies and the low competitiveness in the markets. The environmental values and the conservation of natural resources water, soil and forest- are characteristic positive factors of the production units considered in this study.

\section{KEY WORDS}

Ecological variables, economical variables, socio-cultural variables, cleaner production, sustainability.

\section{INTRODUCCIÓN}

El enfoque de Producción Más Limpia -PML- ha logrado importantes avances en países desarrollados relacionados con la prevención de la contaminación, la eficiencia en el uso de recursos naturales y la minimización de residuos, logros establecidos a partir de la investigación, la planeación, la capacitación, la educación ambiental y la creación de instrumentos económicos y regulatorios claves. Por su parte, en países en desarrollo, se han identificado algunos factores comunes que pueden restringir la aplicación de estrategias de PML, entre los cuales se destacan: "la falta de información sobre el estado actual de la calidad ambiental y la falta de claridad sobre cómo reestructurar los procesos de producción, para lograr incrementar la productividad al tiempo que se reducen los impactos ambientales" (Minambiente, 1997, pag 35). Así mismo, se ha justificado la lentitud de la reconversión productiva hacia una mejor gestión ambiental por:

[...] la resistencia al cambio como un problema cultural y por la dificultad de acceso a la información y a la financiación; igualmente el enfoque hacia mercados locales reduce las exigencias ambientales que pueden presentarse en las exportaciones hacia mercados globales. (CNPMLTA-CECODES, 2000, p. 2)

Estudios realizados por investigadores nacionales e internacionales (Mora et al., 2011; Villanueva et al., 2011) demuestran la importancia del conocimiento local en el contexto 
rural, con el fin de analizar las estrategias de manejo en los sistemas productivos:

\begin{abstract}
Muchos de los recursos a nivel local son desaprovechados tanto por desconocimiento como por lo que los mismos productores llaman inmediatismo, es decir, el reconocimiento práctico que muchos campesinos tienen de que con el uso de insumos químicos se disminuye de manera espectacular el tiempo y el esfuerzo que se hace necesario destinar para ciertas labores del cultivo: desyerba, abono, control de plagas y enfermedades. (Flórez, 2011, p. 28)
\end{abstract}

Un factor importante en el momento de promover estrategias de producción más limpia en el sector agropecuario tiene que ver con la valoración de los indicadores de sostenibilidad asociados a estos procesos, la cual facilita a todos los actores involucrados en el tema (organizaciones no gubernamentales, corporaciones ambientales, entidades públicas y privadas, asociaciones de productores, certificadoras, instituciones académicas) ajustar y enriquecer conjuntamente los procesos de planeación regional y nacional de la sostenibilidad en este sector.

El enfoque participativo en la identificación de buenas prácticas ha evolucionado gracias a los esfuerzos por mejorar el desarrollo y la difusión de tecnologías. Urrego (1989, p. 38) anota que "se dan altos niveles de adopción que no necesitan acciones de transferencia cuando las soluciones tecnológicas, corresponden a las expectativas de los productores". Incluir a los productores en los diagnósticos para la toma de decisiones, debe constituirse en una estrategia para lograr la anhelada sostenibilidad, reflejada en el equilibrio de factores ecológicos, económicos y socioculturales.

En este sentido, los indicadores de sostenibilidad valorados por los productores, así como los procesos de planeación participativos pueden favorecer el uso sostenible de los recursos, en pro del bienestar de los productores: "La planificación participativa de fincas constituye una herramienta básica para incrementar la producción, mejorar el bienestar de la familia y conservar los recursos naturales" (Mora e Ibrahim 2005, p. 5); teniendo en cuenta que "el manejo de los recursos naturales es permeado permanentemente por la racionalidad de las familias productoras, las prácticas culturales que realiza, los objetivos de su producción, las restricciones ambientales de su entorno y las oportunidades de mercado" (Calderón et al., 2011, p. 34), la identificación de los indicadores de sostenibilidad por cada uno de los miembros del hogar no solo permite una buena planificación, sino que también facilita el proceso de apropiación y sentido de pertenencia por su entorno, lo que puede garantizar un mejoramiento continuo y sostenible en el tiempo.

En el proyecto denominado "Evaluación de los beneficios socioambientales generados por estrategias de producción más limpia implementadas en unidades agropecuarias de la cuenca 
media del río Chinchiná", realizado en el marco de la línea de investigación en Saneamiento, Desarrollo y Gestión Ambiental del Grupo de Investigación en Desarrollos Tecnológicos y Ambientales -GIDTA- de la Universidad Católica de Manizales, se planteó como objetivo específico: Establecer indicadores de sostenibilidad para la verificación de la calidad ambiental de sistemas de producción agropecuaria que han implementado estrategias de producción más limpia.

Los indicadores permiten medir una situación específica, con el fin de analizar su evolución a lo largo del tiempo. De acuerdo con los planteamientos de Quiroga (2009, p. 24):

[...] la existencia de problemas ambientales y de sostenibilidad del proceso de desarrollo exige intervenciones y políticas públicas basadas en evidencia e información clara y transparente. De ahí la importancia de concentrar esfuerzos y recursos en la producción de información seleccionada, priorizando aquella que sea clave para mejorar la eficacia de las decisiones. Los indicadores son una buena herramienta para esta tarea, ya que permiten sintetizar información sobre una realidad compleja y cambiante.

Para establecer los indicadores se caracterizaron los sistemas de producción y se consideraron variables económicas, ecológicas y socioculturales, las cuales fueron valoradas por los productores a través de un instrumento que permitió conocer el estado de sostenibilidad en que se encuentra su sistema productivo, a la vez que permite generar un insumo importante para los procesos de planificación predial.

\section{MÉTODOS Y MATERIALES}

A partir de la identificación de seis unidades de producción agropecuaria ubicadas en la cuenca media del río Chinchiná en los municipios de Villamaría, Chinchiná, Manizales y Palestina que han integrado estrategias de PML en sus procesos productivos, se determinaron los indicadores de sostenibilidad para la verificación de la calidad ambiental de los sistemas de estudio.

Estos indicadores basados en la propuesta ASPROINCA (Arango, 2003), se refieren a la aplicación de criterios para medir el grado de sostenibilidad a nivel económico, ecológico y sociocultural que presenta el sistema de producción, mediante la asignación de valores en una escala de 1 a 5, donde al final de la aplicación de cada indicador, aparece un sistema de alarma por colores, que alerta sobre los puntos críticos. Posteriormente se obtiene una gráfica en forma de redegrama ${ }^{3}$, que indica las debilidades y fortalezas desde el punto de vista de la sostenibilidad, la cual permite además de conocer los beneficios ambientales y sociales generados por las estrategias 
implementadas, priorizar acciones que contribuyan a una planificación acertada.

Al evaluar, bajo los mismos parámetros luego de un tiempo, se notará dónde se ha acertado, dónde se ha retrocedido o detenido y qué aspectos hay que potencializar.

Las variables consideradas para cada indicador fueron:

Indicadores económicos: dentro de los indicadores económicos se tuvieron en cuenta la seguridad alimentaria, el mercado, la dependencia de insumos externos y la mano de obra.

Indicadores ecológicos: dentro de estos indicadores se consideraron la conservación del bosque, conservación de los suelos, nivel de complejidad del sistema (el cual está asociado a la capacidad que tiene la finca de tener diferentes sub-sistemas), el nivel de reciclaje y el nivel de integración del sistema.

Indicadores socioculturales: dentro de estos indicadores se tuvo en cuenta la integración familiar, las formas organizativas de trabajo y los valores ambientales los cuales se espera que se transmitan de generación en generación en la familia.

La explicación de cada indicador se presenta en el Anexo 1: Aplicativo Excel de Indicadores de Sostenibilidad.

Con el fin de propiciar el acercamiento con los productores, obtener información y facilitar la selección de las unidades agropecuarias a evaluar, se realizaron conversaciones con el Servicio de Extensión de la Federación Nacional de Cafeteros de Colombia -FNCC-y Unidades Municipales de Desarrollo Rural UMDP-.

Los predios fueron seleccionados considerando los siguientes criterios:

- Implementación de estrategias de producción más limpia.

- Diversificación de la producción. - Propietarios con cualidades de liderazgo comunitario.

- Propietarios con disposición a participar del proyecto y ser evaluados.

El trabajo de campo incluyó: visitas de reconocimiento de las fincas y las estrategias de producción más limpia implementadas, así como la aplicación de instrumentos para la recolección de la información como es el caso del aplicativo de Excel para medir los indicadores de sostenibilidad (Figura 1). 


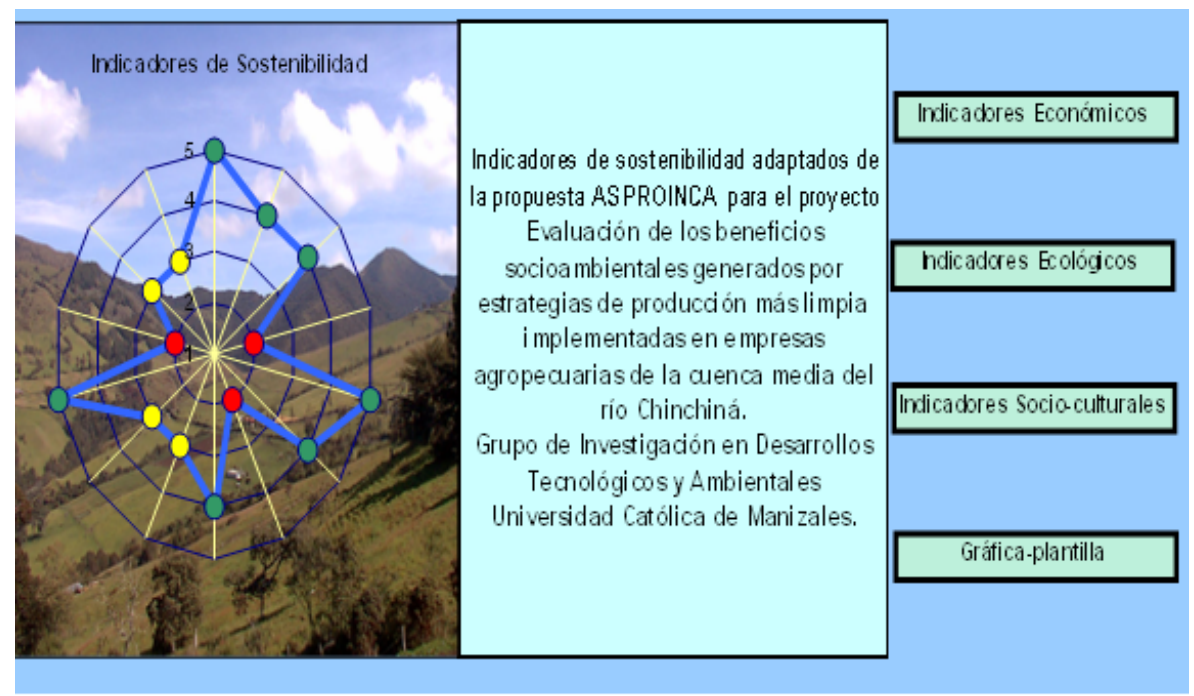

Figura 1. Inicio aplicativo Excel Indicadores de Sostenibilidad. Haga click sobre la imagen para ampliarla

Una vez analizada la información de los predios que cumplían con los criterios establecidos para el proyecto, fueron seleccionados los siguientes: finca Villa Rosa (vereda El Rosario, Manizales), Asociación Rural Mujer Agente de Cambio (vereda Nuevo Río Claro, Villamaría), finca La Aurora (vereda El Pindo, Villamaría), finca San Ignacio ${ }^{4}$ (vereda El Guacamayo, Chinchiná), Granja Agroecológica El Albergue Azul (vereda Alto del Naranjo, Manizales), hacienda Guayabal (vereda Guayabal, Chinchiná), cada uno con diferentes sistemas de producción.

\section{RESULTADOS Y DISCUSIÓN}

\section{1) Finca San Ignacio, vereda El Guacamayo, municipio de Chinchiná}

La finca San Ignacio está localizada en la vereda El Guacamayo, municipio de Chinchiná (Caldas) a 1590 msnm. La familia AlzateEcheverri está conformada por la señora Marta Elena Echeverri y su esposo José Orlando Alzate, quienes son propietarios de la finca desde el año 2008. La finca cuenta con un área de 10,5 cuadras y su sistema de producción principal es el cultivo de café variedad Colombia y el plátano Dominico Hartón. La mano de obra es familiar y contratada, contando con asistencia técnica del Comité de Cafeteros, y capacitaciones según demanda de la comunidad por parte de la Central Hidroeléctrica de Caldas CHEC-y el Servicio Nacional de Aprendizaje -SENA-.

Este predio, se caracteriza porque sus habitantes poseen valores ambientales y liderazgo comunitario, lo que se evidencia en la diversificación agropecuaria del sistema, las buenas prácticas, la conservación de recursos naturales, la integración de subsistemas de producción, el trueque de productos y la recuperación de especies agrícolas con valor nutricional para la seguridad alimentaria de la familia y la comunidad. En esta finca 
se han implementado estrategias de producción más limpia relacionadas con la conservación de recursos naturales y la reducción de materias tóxicas y desechos.

Con respecto a los indicadores de sostenibilidad, la mayoría de los indicadores se encuentran en un nivel alto (valor entre 4 y 5), sin embargo, uno de los aspectos por mejorar en la finca como se visualiza en la siguiente figura, es la alta dependencia de insumos externos y la articulación con el mercado de productos diferentes al café y al plátano, indicadores que se encuentran en niveles medio bajo (valores 2 y 3 , respectivamente) (Figura 2).

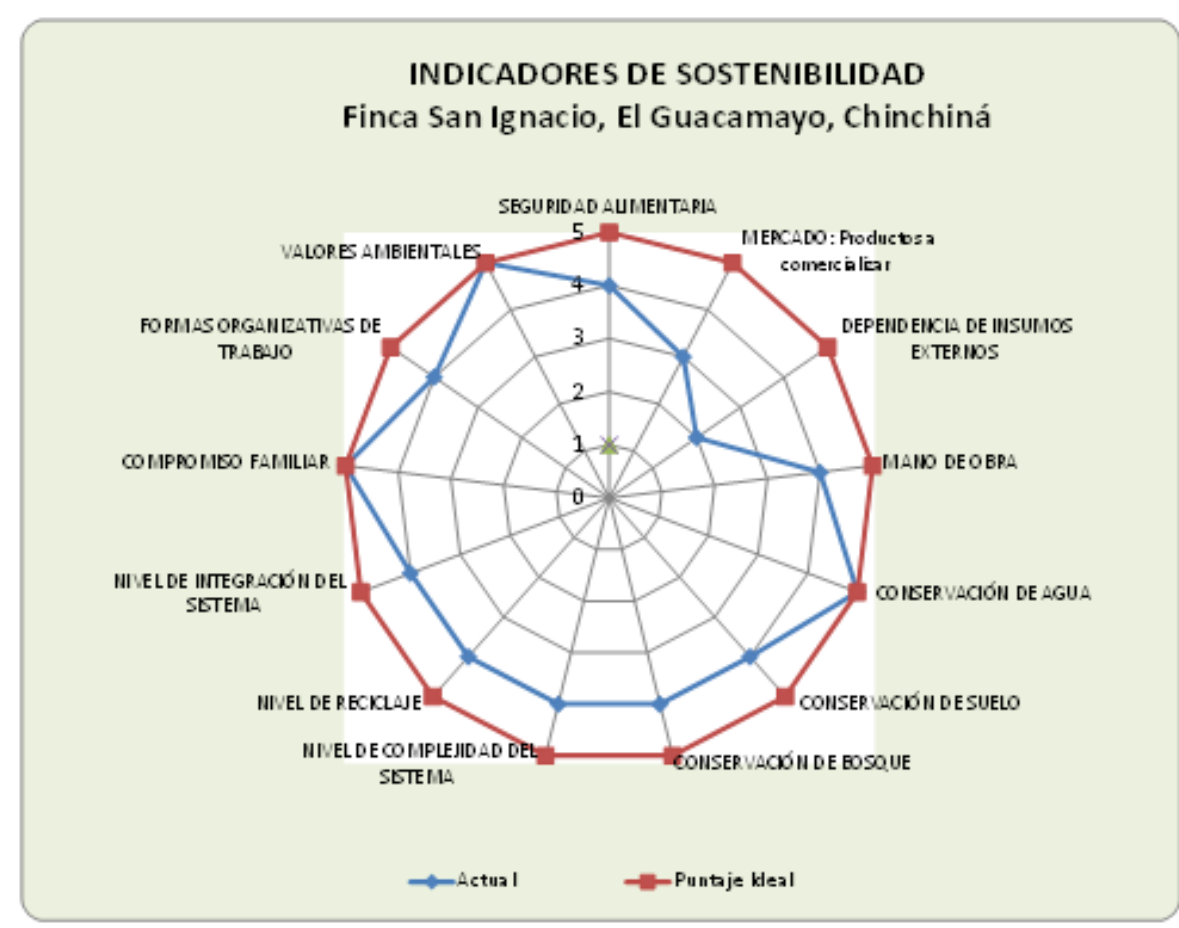

Fuente: Los autores.

Figura 2. Indicadores de Sostenibilidad. Finca San Ignacio. Haga click sobre la imagen para ampliarla

\section{2) Hacienda Guayabal, vereda Guayabal, municipio de Chinchiná}

La hacienda Guayabal está localizada en la vereda Guayabal, municipio de Chinchiná (Caldas) a 1380 msnm. La señora María Teresa Londoño heredó la finca de 83 cuadras, la cual es propiedad de su familia desde hace 58 años. Los principales sistemas de producción de la finca son el café y el agroturismo.

La mano de obra es familiar y contratada, contando con asistencia técnica del Comité de Cafeteros, y capacitaciones según demanda de la comunidad por parte de la Fundación Manuel Mejía y el SENA. 
La hacienda Guayabal del municipio de Chinchiná se dedica al agroturismo asociado al sistema de producción cafetero, donde se han implementado estrategias de PML orientadas principalmente hacia la conservación de los recursos naturales: agua, suelo, biodiversidad. Esta hacienda ha tenido avances significativos en su sistema productivo, en la aplicación de buenas prácticas agrícolas y en la transformación tecnológica del proceso del café, así como en la implementación de buenas prácticas en el servicio de turismo.

Frente a la valoración de los indicadores de sostenibilidad, los que se encuentran en un nivel medio (valor 3) son los relacionados con: la dependencia de insumos externos, el mercado, la seguridad alimentaria, el nivel de integración del sistema, nivel de complejidad del sistema y las formas organizativas del trabajo, considerándose que estos son los aspectos más importantes a tener en cuenta en el momento de proyectar las posibles mejoras de la finca en el proceso de planificación predial (Figura 3).

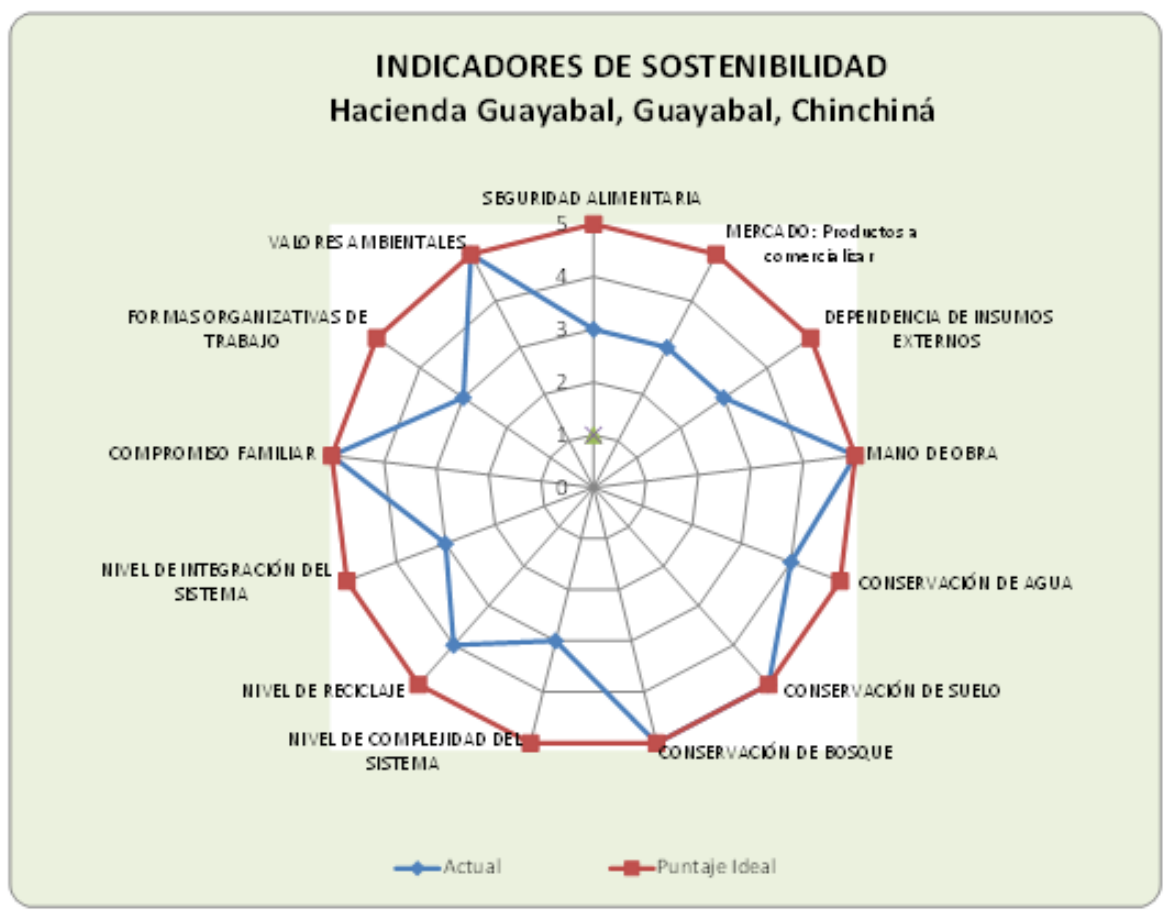

Fuente: Los autores.

Figura 3. Indicadores de Sostenibilidad. Hacienda Guayabal. Haga click sobre la imagen para ampliarla

\section{3) Finca Villa Rosa, vereda El Rosario, municipio de Manizales}

La finca Villa Rosa está localizada en la vereda El Rosario, municipio de Manizales (Caldas) a $1400 \mathrm{msnm}$. La propietaria, María Emilia Jaramillo de Arango, madre del administrador de la finca, Francisco Arango, ha conservado la finca desde hace 80 
años, la cual cuenta con 29 cuadras de café y plátano principalmente.

La mano de obra es familiar y contratada, contando con asistencia técnica de un agrónomo y del Comité de Cafeteros, y capacitaciones según demanda de la comunidad por parte del SENA. Esta finca cuenta con certificación vigente en café sostenible UTZ, la cual consiste en el cumplimiento de requisitos relacionados con mejores prácticas en el sistema de producción, mejores condiciones laborales, uso sostenible de recursos naturales, con un enfoque de mejora continua.

A pesar de contar con la certificación UTZ, algunos aspectos por mejorar en la finca Villa Rosa son la alta dependencia de insumos externos, el nivel de complejidad del sistema, además de fortalecer los valores ambientales, la seguridad alimentaria y diversificar la producción para la venta (Figura 4).

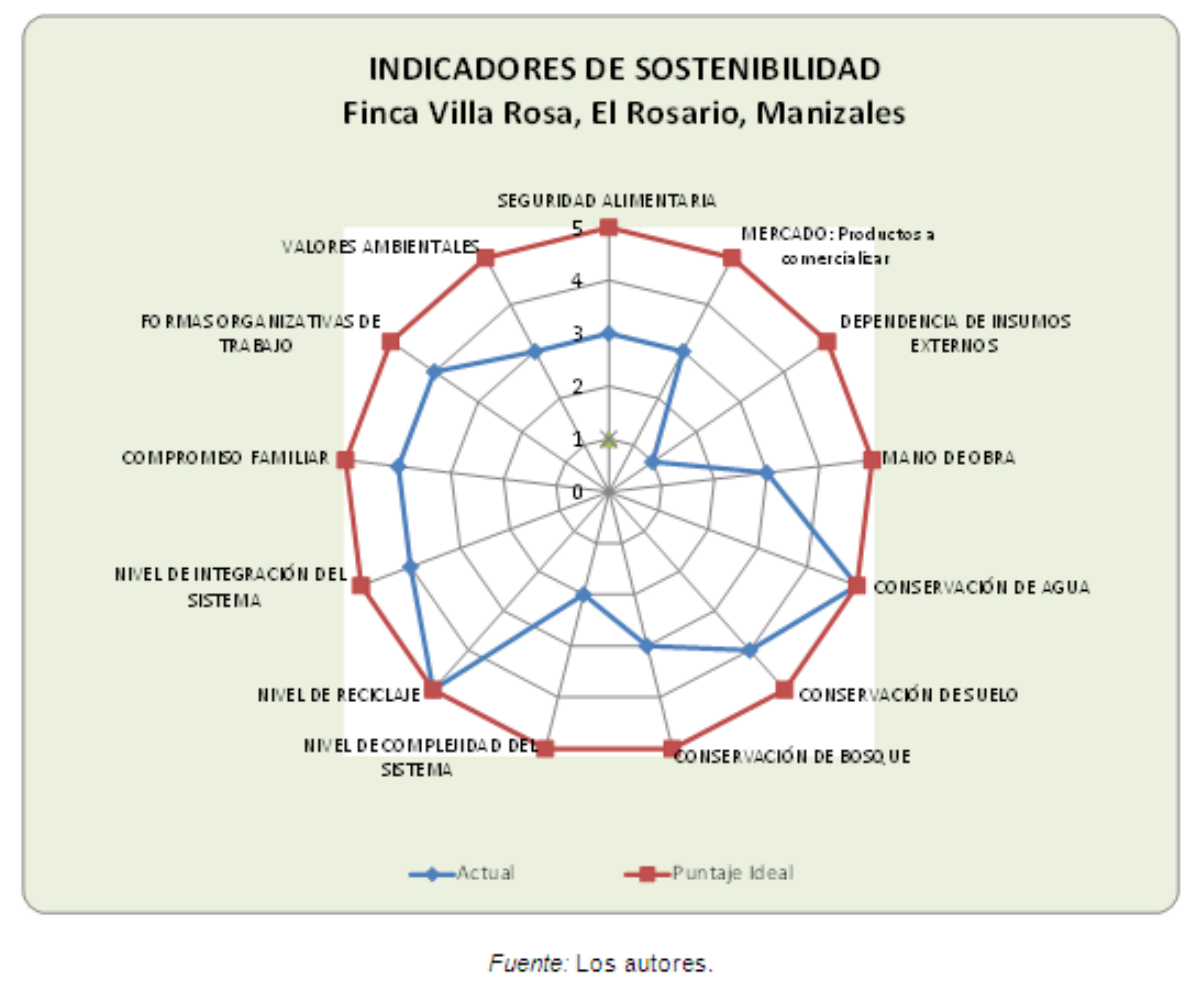

Figura 4. Indicadores de Sostenibilidad. Finca Villa Rosa.

Haga click sobre la imagen para ampliarla

4) Finca La Aurora, vereda El Pindo, municipio de Villamaría

La finca La Aurora está localizada en la vereda El Pindo, municipio de Villamaría (Caldas) a 2400 msnm. El propietario, Francisco Arango, ha conservado la finca desde hace 30 años, la cual cuenta en total con 370 cuadras entre pastos para sistema de producción lechera, reforestación articulada al Proyecto Forestal para la Cuenca del Río Chinchiná -PROCUENCA- y sistema de producción porcícola. 
La mano de obra es familiar y contratada, contando con asistencia técnica de un médico veterinario zootecnista y de la Asociación Nacional de Porcicultores, la cual facilitó la obtención de la Certificación en Buenas Prácticas Ganaderas -BPG- en la producción porcícola que otorga el Instituto Colombiano Agropecuario -ICA- con el fin de dar cumplimiento de la normatividad sobre inocuidad en la producción de carne de cerdo (Resolución 2640 de 2007 expedida por el ICA). Para lograr la certificación la finca debe garantizar la sanidad animal y bioseguridad de la granja a través de plan de vacunación, plan sanitario, plan de emergencias, procedimientos de limpieza y desinfección, manejo ambiental y disposición de mortalidades, gestión de riesgos sanitarios, biológicos y químicos en la producción de carne de cerdo. Además, se cuenta con la generación de bioabono para fertilización orgánica de pasturas a partir de un tanque estercolero.

Esta finca cuenta con un área total destinada a la conservación y reforestación de 99 cuadras de relictos de bosque, plantaciones forestales, cercas vivas, linderos maderables con especies maderables, forrajeras y de servicios ecosistémicos como: pino, eucalipto, aliso, encenillo, yarumo, drago, cedro negro, botón de oro y tilo.

A pesar de contar con la certificación en BPG, algunos aspectos por mejorar en la finca La Aurora son la alta dependencia de insumos externos, la seguridad alimentaria, la diversificación de la producción para la venta, además de fortalecer los valores ambientales y el nivel de complejidad del sistema (Figura 5).

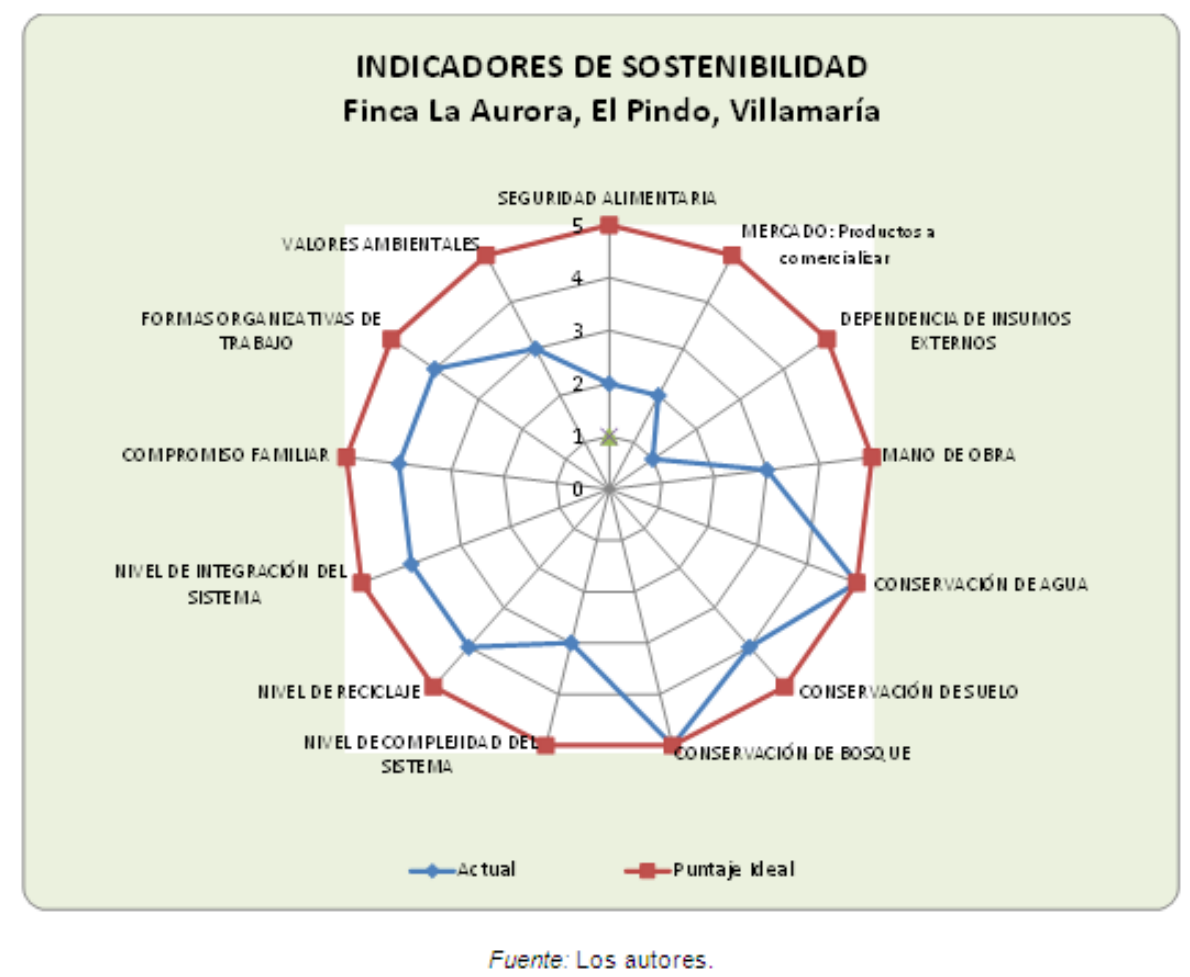

Figura 5. Indicadores de Sostenibilidad. Finca La Aurora. Haga click sobre la imagen para ampliarla 


\section{5) Granja Agroecológica El Albergue Azul, vereda Alto del Naranjo, municipio de Manizales}

La Granja Agroecológica El Albergue Azul está localizada en la vereda Alto del Naranjo, municipio de Manizales (Caldas) a 1800 msnm. La propietaria, señora Cecilia Rivas, vive en la finca con su esposo desde el año 1987. Este predio tiene un área de 1,7 hectáreas, dedicadas a la filosofía de "protección de la biodiversidad".

La mano de obra es familiar, contando con asistencia técnica del Comité de Cafeteros, y capacitaciones según demanda por parte de Aguas de Manizales, y el Jardín Botánico de la Universidad de Caldas. Esta finca contó con certificación de producción ecológica ECOCERT, aunque actualmente no está vigente por altos costos de la refrendación.

La familia Acevedo-Rivas se caracteriza por la tradición campesina, por los conocimientos y la experiencia en producción agroecológica, así como por la promoción de procesos asociativos y educativos en la vereda.

El sistema productivo es totalmente orgánico, el cual favorece la conservación de biodiversidad y semillas de especies tradicionales, uso sostenible de recursos naturales, diversificación de la producción agropecuaria, mantenimiento de huerta casera para autoconsumo y venta de excedentes.

Asimismo, la granja se beneficia del Convenio World-Wide Opportunities on Organic Farms (WWOOF) por el cual frecuentemente se cuenta con la visita de turistas extranjeros, con los cuales se realiza un trueque de alojamiento y alimentación por aprendizaje a partir de trabajo en la granja. Además, se obtienen ingresos económicos por la venta de café marca El Albergue Azul.

Con respecto a los indicadores de sostenibilidad, se puede observar el excelente desempeño socio-ambiental de la granja. Se deben mejorar los aspectos relacionados con la economía del sistema productivo (Figura 6). 


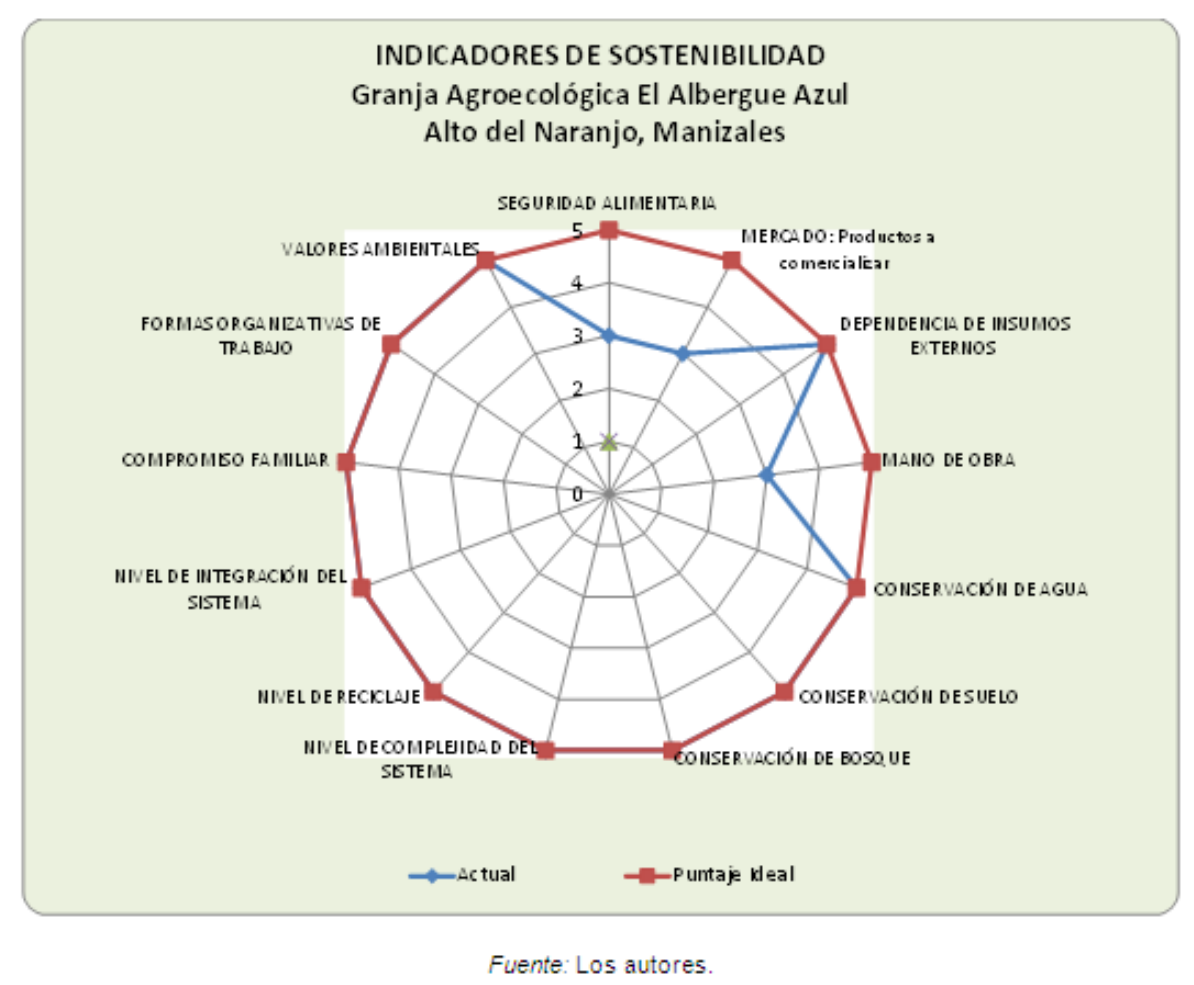

Figura 6. Indicadores de Sostenibilidad. Granja Agroecológica El Albergue Azul. Haga click sobre la imagen para ampliarla

\section{6) Asociación Mujer Rural Agente de Cambio, vereda Río Claro, municipio de Villamaría}

Esta asociación, liderada por la señora Beatriz Sierra Duque, se encuentra ubicada en la vereda Río Claro del municipio de Villamaría (Caldas). Las mujeres que integran AMRAC se dedican a la producción agroecológica de tomate y otros productos de pancoger como maíz y fríjol. Las estrategias de producción más limpia implementadas están relacionadas con sistemas agroforestales, disminución de residuos tóxicos y manejo de residuos sólidos.

La Asociación Mujer Rural Agente de Cambio está conformada por 25 miembros. Se obtuvo la personería jurídica desde junio de 2012 e inició actividades con la capacitación del SENA sobre producción de tomate, para lo cual se realizó el montaje del invernadero en la casa de la presidente de la Asociación. Por falta de experiencia de las miembros de la asociación hubo pérdida en la primera cosecha y debido a que no hubo remuneración económica, y a la característica de quienes conforman la asociación: mujeres cabeza de hogar, esta se debilitó y en la actualidad solo se cuenta con seis personas activas en el cultivo como tal, el cual se rota con cultivo de habichuela.

En la mayoría de los patios de las casas se cuenta con pequeñas huertas en las que se cultiva maíz, fríjol, cilantro, cebolla larga, yuca, arracacha, mafafa, entre otras hortalizas. 
Se considera que se cuenta con un uso sostenible del suelo, ya que se cuenta con prácticas como la incorporación de abonos orgánicos basados en gallinaza, pulpa de café y residuos de cocina.

Con respecto a los indicadores de sostenibilidad, las variables económicas, ecológicas y socioculturales se encuentran en un nivel medio y bajo, debido al proceso incipiente de la asociación, que aunque cuenta con valores ambientales, las circunstancias sociales no favorecen los demás aspectos (Figura 7).

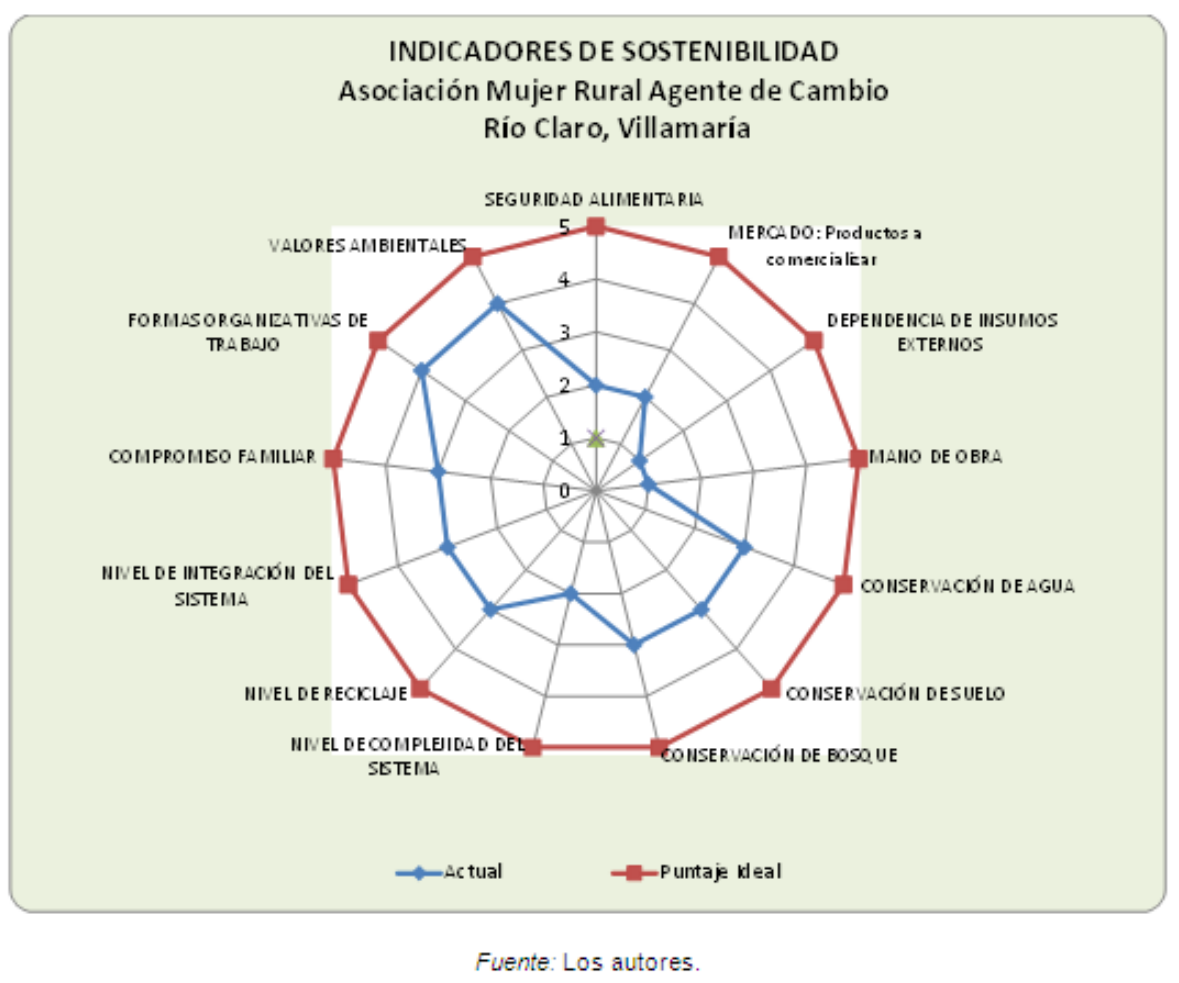

Figura 7. Indicadores de Sostenibilidad. Asociación Mujer Rural Agente de Cambio. Haga click sobre la imagen para ampliarla

\section{CONCLUSIONES}

La metodología de indicadores adaptada en este estudio para la generación de redegramas a partir de la valoración de indicadores de sostenibilidad en cada unidad productiva, permite la visualización del estado de los predios en términos de sostenibilidad, a la vez que facilita los procesos de planificación.

En la mayoría de los predios uno de los aspectos por mejorar es el relacionado con la alta dependencia de insumos externos, esto se debe a que aún se siguen aplicando insumos químicos, especialmente en momentos de presencia de plagas $y$ enfermedades de alta incidencia y fácil propagación. Los insumos externos también incluyen los alimentos comprados, debido al fenómeno de falta de huertas en la mayoría de las fincas. 
En la Asociación Rural Mujer Agente de Cambio, se obtuvo el menor nivel de valoración en la mayoría de los indicadores de sostenibilidad, situación que se hace comprensible dado que apenas se está iniciando el proceso de implementación de estrategias de producción más limpia.

La conservación del agua, es el indicador ecológico que presenta mayor nivel de valoración en la mayoría de los predios, esto se debe a que hay una alta sensibilización y cultura de cuidado de este recurso natural.

El mercado es un indicador económico que presenta en general bajos niveles de valoración, los propietarios manifiestan que aún no existe disposición de pagar por parte de los consumidores para adquirir un producto inocuo, lo que hace que los productos agropecuarios generados a partir de un proceso agroecológico y de buenas prácticas, no tengan un precio especial por su valor agregado.

En cuanto a la seguridad alimentaria son pocos los predios donde se puede asegurar permanentemente, en áreas específicas como las huertas o asociadas al sistema productivo, una base alimentaria que incluya los productos agrícolas y productos pecuarios que garantice la alimentación de la familia y la producción de excedentes (incluyendo forrajes) para la alimentación de los animales.

Para disminuir los riesgos y asegurar un flujo permanente de ingresos, los predios deben proveer una variedad de productos que se comercialicen, en este sentido, a mayor número de productos mejor será la situación del productor.

Una finca presenta mayor grado de sostenibilidad si hay una baja dependencia de insumos externos, los cuales pueden estar representados en compra de insumos agrícolas y pecuarios, alimentos, materiales para construcción y otros.

La sostenibilidad de las estrategias de PML permanecerá en la familia siempre que se recuperen, mantengan y transmitan los valores ambientales que se reflejen en las actividades que realiza la familia en su sistema productivo.

Es importante anotar que este estudio tendrá continuidad a partir del proyecto iniciado en el año 2014: "Análisis de Estrategias de Producción más Limpia en el Sistema de Producción del Plátano (Musa spp.), en el municipio de Manizales".

\section{AGRADECIMIENTOS}

Las autoras presentan sus agradecimientos a los productores propietarios de las fincas de estudio: Beatriz Sierra (Nuevo Río Claro, Villamaría), Cecilia Rivas (Alto del Naranjo, Manizales), Marta Elena Echeverri y José Orlando Alzate (El Guacamayo, 
Chinchiná), María Teresa Londoño (Guayabal, Chinchiná) y Francisco Arango (El Pindo, Villamaría; El Rosario, Manizales) por su participación activa en el proyecto. Al Servicio de Extensión del Comité Departamental de Cafeteros de Caldas y a las Unidades Municipales de Desarrollo Rural de Manizales y Chinchiná por la asesoría brindada. Al Centro de Investigación, Proyección y Desarrollo de la Universidad Católica de Manizales, por la financiación del estudio y las gestiones realizadas para la ejecución satisfactoria del proyecto.

\section{POTENCIAL CONFLICTO DE INTERESES}

No hay conflicto de intereses.

\section{FUENTES DE FINANCIACIÓN}

Este artículo es resultado del proyecto "Evaluación de los beneficios socioambientales generados por estrategias de producción más limpia implementadas en unidades agropecuarias de la cuenca media del río Chinchiná", realizado en el marco del Grupo de Investigación en Desarrollos Tecnológicos y Ambientales -GIDTA- durante los años 20122013 y financiado por el Centro de Investigación, Proyección y Desarrollo de la Universidad Católica de Manizales.

\section{REFERENCIAS}

- Arango, H. (2003). Planificación predial participativa. CD-ROOM. Fundación Centro para la Investigación en Sistemas Sostenibles de Producción Agropecuaria, CIPAV, Cali, Colombia.

- Calderón, P.A. et al. (2011). Planificación participativa de fincas de aprendizaje: el caso de Colombia. En Cristóbal Villanueva, Claudia J. Sepúlveda y Muhammad Ibrahim (Eds.). Manejo agroecológico como ruta para lograr la sostenibilidad de fincas con café y ganadería (pp. 29-50). 1 ed. Turrialba, CR: CATIE.

- CNPMLTA-CECODES. (2000). Producción más limpia en Colombia. Conceptos sobre motivaciones y obstáculos para su implementación en Colombia. Medellín, Colombia. 12 p.

- Flórez, D.R. (2011). La transformación productiva de la finca campesina. Respuesta de los productores a la crisis cafetera. En Jairo Mora Delgado et al. Medios de vida y materiales orgánicos en fincas campesinas (pp. 23-37). Ibagué: Universidad del Tolima, Red Alma Máter.

- Ministerio de Medio Ambiente. (1997). Política Nacional de Producción más Limpia. Bogotá: Minambiente pag 35.

- Mora, J. et al. (2011). Medios de vida y materiales orgánicos en fincas campesinas. Ibagué: Universidad del Tolima, Red Alma Máter. 
- Mora Delgado, J. e Ibrahim, M. (2005). Diversificación de fincas pecuarias: estrategia de vida para aliviar la pobreza rural. Memorias de Conferencia Electrónica. LEAD-FAO-CATIE Turrialba Costa Rica. Formato html, 60 kb. 17p. Recuperado el 31 de marzo de 2006 de http://www.virtualcentre.org/es/ele/conferencia4/síntesis.htm

- Quiroga, Rayén. (2009). Guía metodológica para desarrollar indicadores ambientales y de desarrollo sostenible en países de América Latina y el Caribe. Serie Manuales 61. CEPAL.

- Urrego, M.G. (1989). El enfoque de sistemas de producción como estrategia para mejorar niveles de adopción de tecnología. Boletín técnico No. 198. Bogotá: Instituto Colombiano Agropecuario.

- Villanueva, C. Sepúlveda, C. Ibrahim, M. (Eds) (2011) Manejo agroecológico como ruta para lograr la sostenibilidad de fincas con café y ganadería. 1 ed.-Turrialba, CR: CATIE, 243 p.

1. Administradora Ambiental. Magíster en Sistemas de Producción Agropecuaria. Docente investigadora. Líder Grupo de Investigaciones en Desarrollos Tecnológicos y Ambientales. Universidad Católica de Manizales. pcalderon@ucm.edu.co

2. Administradora Ambiental. Magíster en Desarrollo Sostenible y Medio Ambiente. Estudiante de Doctorado en Desarrollo Sostenible de la Universidad de Manizales. Docente investigadora, Universidad Católica de Manizales. gyflorez@ucm.edu.co

3. El redegrama es un gráfico radial que muestra diferentes valores con relación a un punto inicial. Para este caso, le permite al propietario del predio reconocer las debilidades y fortalezas de su unidad productiva respecto a temas económicos, ecológicos y socioculturales, identificando puntos críticos para la priorización de actividades de sostenibilidad.

4. Cabe anotar que el predio San Ignacio, no hace parte de la cuenca del río Chinchiná, pero se consideró para este proyecto por presentar características especiales de sostenibilidad, en términos ecológicos, económicos y socioculturales, además por la dificultad de encontrar predios dentro de la cuenca con las características necesarias para hacer parte del proyecto.

Para citar este artículo: Calderón Cuartas P.A. \& Flórez Yepes G. Y. (2014). Valoración y análisis de indicadores de sostenibilidad en seis unidades de producción agropecuaria de la cuenca media del río Chinchiná. Revista Luna Azul, 41, 73-88. Recuperado de http://lunazul.ucaldas.edu.co/index.php?option=content\&tas k=view\&id=1056 